\title{
Ubiquitous Information Retrieval Using Multi-level Characteristics
}

\author{
Joonhee Kwon ${ }^{1}$ and Sungrim $\mathrm{Kim}^{2}$ \\ ${ }^{1}$ Department of Computer Science, Kyonggi University, \\ San 94-6, Yiui-dong, Yeongtong-ku, Suwon-si, Kyonggi-do, Korea \\ kwonjh@kyonggi.ac.kr \\ ${ }^{2}$ Department of Computer Science, Seoil College, \\ 49-3, Myonmok-dong, Jungrang-Ku, Seoul, Korea \\ srkim@seoil.ac.kr
}

\begin{abstract}
Applications of ubiquitous computing are increasingly leveraging contextual information from several sources to provide users with behavior appropriate to their environment. The method of information retrieval is one of the most fundamental research issues in ubiquitous computing. Applications where user's contexts change continuously over time require prompt retrieval of relevant information. This paper proposes a new ubiquitous retrieval method that enables users to obtain relevant information efficiently using the multi level characteristics of the contexts. This paper describes the ubiquitous information retrieval process. Several experiments are performed and the results verify that proposed method has better retrieval performance.
\end{abstract}

\section{Introduction}

Weiser introduced the area of ubiquitous computing, and put forth a vision of people and environments augmented with computational resources that provide information and services whenever and wherever desired [1]. One of the most critical technologies in a variety of application services of ubiquitous computing is to supply adequate information or services depending on each context through context-awareness. The context is characterized by being continuously changed and defined as all information related to the entities such as users, space and objects [2,3]. Ubiquitous computing applications need to be context-aware, adapting behavior based on information sensed from the physical and computational environment.

According to recent analysis on applications using context-aware ubiquitous computing, one of the most challenging research areas in the future is considered to be information retrieval methods in ubiquitous computing environment $[4,6]$. The main consideration in information retrieval methods is how accurately it retrieves information required by the users and how rapidly it retrieves information even with the bulk of information to retrieve [4, 7]. Since existing ubiquitous computing retrieval methods get all detailed information for the target context in accordance with the continuous changes of contexts, the retrieval time takes longer. Moreover, since the retrieval results could lead to unwanted information for users, there is the problem in retrieving accurate information. Thus, new retrieval methods are required for rapid and accurate information retrieval.

We suggest a new ubiquitous retrieval method using multi level characteristics of contexts for rapid and accurate information retrieval. We adopt a multi level charac- 
teristic of contexts based on the observation that all information does not need to be retrieved at a time according to each context value. By using the multi level characteristics of contexts, we get more detailed information progressively as we get nearer to the context of interest. This enables more rapid and accurate information retrieval because of no access to the most detail information in the context values at a time.

Our discussion will proceed as follows. Section 2 will give an overview of related works. Section 3 will discuss the ubiquitous information retrieval process and section 4 will discuss the experimental results. Finally section 5 will conclude this paper.

\section{Related Works}

Most approaches to context-aware retrieval methods simply match user profiles to context values [6]. Because the mechanisms use only explicit information, there are limitations to retrieve useful information. Moreover, the method does not consider retrieving information rapidly.

An advanced approach to context-aware retrieval method in ubiquitous computing is proposed in [4]. It proposes a context-aware cache based on the context-aware diary. Based on the contents, the context-aware cache tries to capture the information the user is more likely to need in future contexts. It makes more immediate retrieval and reduces the cost of retrieving information by doing most of the work automatically. The context-aware diary stores past and future data that are explicitly informed. This approach, however, simply matches data from the context-aware diary to the current context, when the context-aware cache tries to capture future data.

The most recent approach to context-aware retrieval method is proposed in [8]. It uses a very similar approach to the approach we have taken. To retrieve information rapidly, this method locally stores the recommended information that the user is likely to need in the near future based on behavior patterns. To retrieve the information that would be required by the user, this method uses data mining methods. Moreover, using a multi-agent architecture, it allows continuous rapid and accurate information retrieval even with the change of user's contexts and solves the limitations of storage. However, this method retrieves the most detail information at a time causing unnecessary and slow information retrieval. Moreover, when the behavior pattern is not found, the method does not retrieve information rapidly and accurately.

The largest difference between our work and [8] is adoption of the multi level characteristics of context. It may reduce the amount of information requested for faster retrieval time and retrieve more accurate information for users.

\section{Ubiquitous Information Retrieval Process}

The proposed method for information retrieval is conceptually comprised of three main tasks, as shown in Figure 1. The first step is to extract the recommendation information rules related to the context values [8]. These are extracted using the association rule mining in the data mining [9] methods from the contexts database.

In the second step, the recommendation information in the near future is extracted using the current context value and recommendation information rules. In this paper, we call the window includes context values that have possibility of using in near future as "context window". When a behavior pattern [8] is found, the context values in 


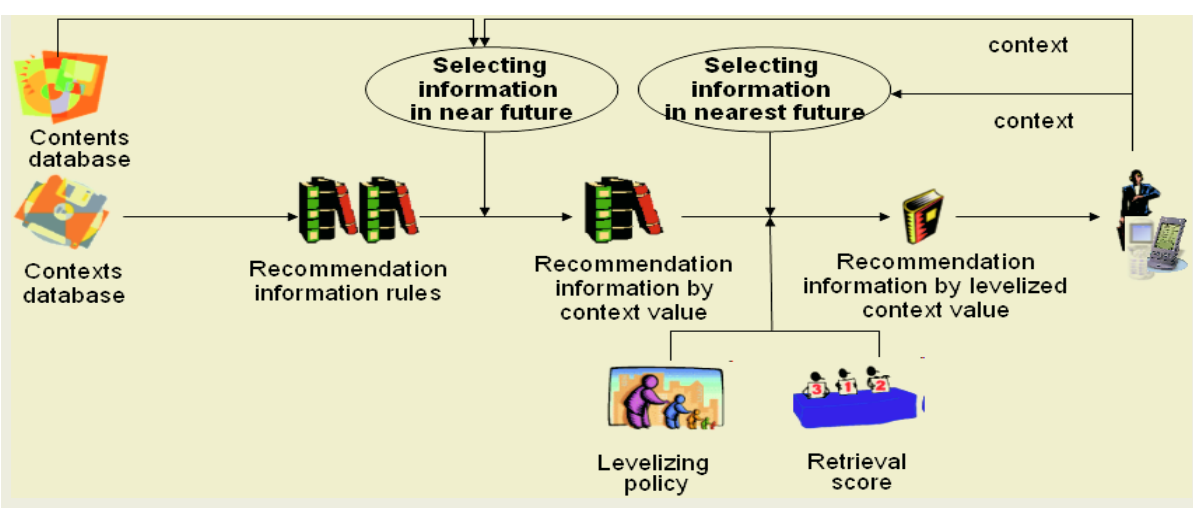

Fig. 1. Flow Diagram of the Proposed Method

context window is a behavior pattern, otherwise they are context values within a certain difference of value from the current context values.

In the final step, we extract the recommendation information to be appeared in the very near future using context value, levelizing policy and retrieval score. The recommendation information extracted in the second step can cause the storage capacity and transmitting rate to increase. To solve these problems, only the recommendation information that can be used in the very near future is stored and then this process is repeated.

The levelizing policy determines the level value of the current context value. In this paper, we assume that the levelizing policy is given. The level value means the level of detail of the information and determines properties of information to be retrieved. The information needed for a low level value is higher in priority and broader than it needed in a high level value. In this paper, we use the confidence in association rule mining as priority. For an example of the levelizing policy, the level value may be determined using the velocity in the location context. That is, faster (slower) velocity is considered as the request for simple (detailed) information on wider (narrower) area so that the level value is lower (higher).

For rapid and accurate information retrieval, we use the prefetching method. From the recommendation information by the second step, we extract recommendation information to be retrieved in the very near future using the current context value and level value. The size of context window in the very near future is determined by the level value and becomes larger in inverse proportion to the level value. We only extract information higher than priority allowed in current level value from the recommendation information of context values in the context window.

However, a replacement policy is needed by storage capacity limitation. Therefore, we propose the retrieval score. The retrieval score represents the priority of information determined by a level value of a context value when the context values are in the context window. The higher the retrieval score of information is, the higher the possibility to be accessed in the very near future is. We replace the information in the lower retrieval score with it in the higher retrieval score. It makes the high hit ratio in the client's storage. The retrieval score is zero in case the context values are not in the context window. 


\section{Experimental Evaluation}

For experiments, we implemented both the proposed method called System 1 and the method in [8] called System2. All programs were written in Java. The server ran on a Pentium IV desktop computer. The client ran on a Pentium laptop computer.

In the experimental data, the number of context values was set to 100 and the number of recommendation rules was set to the number of context values multiplied by the row number of recommendation information. The number of rows of recommendation information was set to the number of rows of contents multiplied by 0.005 .

The values of the contents were generated from number 1 to the number of the contents. The context values were generated from number 1 to the number of the context values. The action parts of recommendation rules were randomly generated from the values of contents for each condition parts, where condition parts are context values. The confidence of the rules is given randomly from 20 to 100. The number of levels in generated data is 4 . For each level, the size of context window required in level value $1(2,3,4)$ is $10(7,4,1$, respectively) and confidence allowed in level value $1(2,3,4)$ is $80(60,40,20$, respectively). In addition, level values are higher whenever context values are changed in our experiment.

We evaluated the impact of the amount of contents for each level value. We ran an experiment 100 times for context values extracted at random for evaluation. In order to evaluate the impact of the row number of contents, we measured the average miss ratio in client for each context value to retrieve recommendation information. We set the maximum number of row allowed in client's storage to 100. For the comparison, we varied row numbers of contents by $10 \%$ from 10,000 to 31,384 . The measurements of the evaluation for each level value are depicted in Figure 2.

As shown in Figure 2, for System1, the average miss ratio is near zero when the row number of contents decreases. However, average miss ratio for System 2 exceeds $50 \%$ in level value 1, 2 and 3. Only in level value 4, average miss ratio for System 1 and System 2 is almost the same. In level value 4, both System1 and System2 use the most detail information; therefore the results are almost the same. Several other observations are found on these results. First, the average miss ratio in System 1 consistently performs better than that in the System2. Second, as the level value is lower, the difference between the System 1 and System 2 is increased. As the level value is lower, the number of context values required in the near future is greater. The result shows the high miss ratios in System2.

\section{Conclusion}

Ubiquitous information retrieval is very promising but has not been sufficiently explored. This paper introduced a new ubiquitous retrieval method using multi level characteristics of contexts. The proposed method was explained with the system flow, the scenario, the algorithms and the experimental results.

There have been some studies in context-aware retrieval methods for ubiquitous computing. These methods however, all use the most detailed information. We suggest a new ubiquitous retrieval method using the multi level characteristics of contexts for rapid and accurate information retrieval. By the multi level characteristics of contexts, we get more detailed information progressively as we get nearer to the con- 


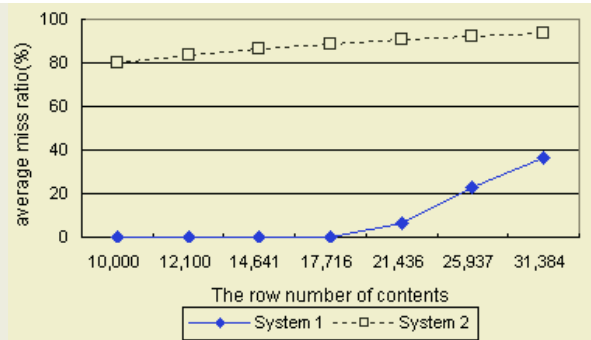

(a) Level 1

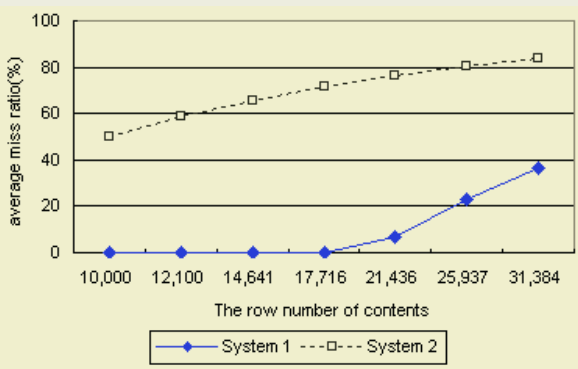

(c) Level 3

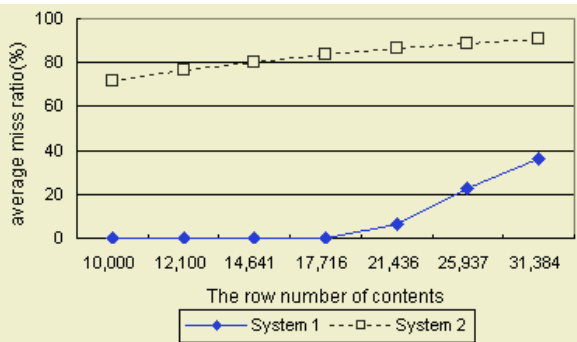

(b) Level 2

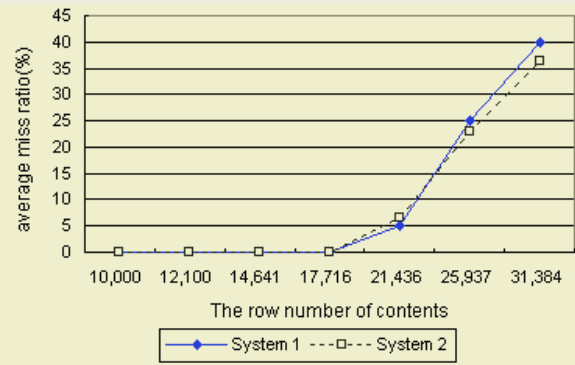

(d) Level 4

Fig. 2. The Impact of the Row Number of Contents

text of interest. This enables the rapid and accurate information retrieval, because of no access to the most detail information in all context value at a time.

The proposed method has several advantages. First, we presented a new ubiquitous information retrieval method using multi level characteristics of contexts. By using these multi level characteristics of contexts, information is retrieved rapidly and accurately. Second, we proposed a new prefetching and replacement method for levelized context-aware information. The proposed method could be used in ubiquitous applications to improve information retrieval.

\section{Acknowledgements}

This work was supported by grant No.R04-2004-000-10056-0 from the Basic Research Program of the Korea Research Foundation.

\section{References}

1. Mark Weiser: The computer for the 21st century. Scientific American, Vol. 265, No. 30, p.94-104, 1991

2. Anind K. Dey, Daniel Salber, Gregory D. Abowd: A Conceptual Framework and a Toolkit for Supporting the Rapid Prototyping of Context-Aware Applications. Anchor article of a special issue on Context-Aware Computing Human-Computer Interaction Journal, Vol. 16, No. 2-4, p.97-166, 2001

3. Paul Prekop, Mark Burnett: Activities, Context and Ubiquitous Computing. Special Issue on Ubiquitous Computing, Computer Communications, Autumn, 2002 
4. P. J. Brown, G. J. F. Jones: Context-aware Retrieval: Exploring a New Environment for Information Retrieval and Information Filtering. Personal and Ubiquitous Computing, 2001, Volume 5, Issue 4, December, p.253 - 263, 2001

5. Jeffrey Hightower, Gaetano Borriello: Location Systems for Ubiquitous Computing. Computer, Vol. 34, No. 8, p 57-66, IEEE Computer Society Press, 2001

6. Getri Kappel, Birgit. Proll, Werner Retschitzegger, Wieland Schwinger: Customisation for Ubiquitous Web Applications - A Comparison of Approaches. International Journal of Web Engineering and Technology, Vol.1, No.1, p. 79-111, January, 2003

7. Bradley J. Rhodes, Pattie Maes: Just-in-time information retrieval agents. IBM Systems Journal, Vol. 39, No. 3\&4, p.685-704, 2000

8. Joonhee Kwon, Sungrim Kim, Yongik Yoon: Just-In-Time Recommendation using MultiAgents for Context-Awareness in Ubiquitous Computing Environment. Lecture Notes in Computer Science 2973, Mar, 2004

9. R. Agrawal, T. Imielinski, A. Swami: Mining association rules in large databases. In Proceedings of ACM SIGMOD Conference on Management of Data, Washington D.C., p.207216, May 1993 